\title{
OUTPUT FEEDBACK CONTROL OF SWITCHED NONLINEAR SYSTEMS: A GAIN SCHEDULING APPROACH
}

\author{
Vojtech Veselý $^{*}$ — Adrian Ilka**
}

\begin{abstract}
Switched controller design for nonlinear continuous and discrete-time systems under an arbitrary switching signal using the gain scheduling approach is addressed in this paper. The obtained controller design procedures for continuous and discrete-time systems are in the bilinear matrix inequality form. The proposed design procedure ensures multi parameterdependent quadratic stability of the switched gain scheduled plant which is associative to a nonlinear plant model and optimal performance defined by quadratic gain scheduled parameters weighting cost function. Example demonstrates the effectiveness of the proposed approaches.
\end{abstract}

K e yw ords: switched controller, non-linear system, gain-scheduled control, controller design, structured controller, Lyapunov function

\section{INTRODUCTION}

Switched systems consist of a set of subsystems and a switching signal selecting a subsystem to be active during a time interval. Switched systems have many applications in many fields of real plant control. There are several approaches to model hybrid systems [16]. In [4] a model of a large class of hybrid systems is given that considers discrete event systems and continuous dynamics modeled by differential or difference equation. Such models are used to formulate a general stability analysis and controller synthesis framework for hybrid systems. Results for modeling and stability analysis of hybrid systems have been presented in $[7,16,17]$.

In this paper we follow the class of hybrid systems known as switched systems [15]. The reader can find a survey of the present state of hybrid systems in the excellent paper [17] and book [16]. Stability and the controller design procedure are the most important issues in the study of switched systems, specially of nonlinear ones. Stability under arbitrary switching is guaranteed by the existence of a common Lyapunov function for all subsystems $[9,14,24,29]$. For switched linear systems, finding the common Lyapunov function is relatively easy but for nonlinear systems it is difficult. A survey of switched nonlinear systems can be found in $[2,14,20,29]$. Due to the switched controller design problems for continuous and discrete time nonlinear systems, in this note we pursue the idea to use - instead of a model of a nonlinear switched plant - a model of the switched gain scheduled plant and to design a switched gain scheduled controller guaranteeing closed loop stability and an optimal value of a given quadratic novel cost function for all operating points of the switched nonlinear system.
A number of papers deal with the design of a switched controller for discrete-time systems $[4,16,17]$. There are only some results in the field of switched (gain scheduled) controller design for continuous-time systems $[10,19,23$, $25]$ and stabilization of switched continuous time linear systems $[3,6]$, where in $[19]$ the switching gain scheduled control technique is used to control the flexible ball-screw drive servo with a wide range of operating conditions. The framework of [25] is based on the linear matrix inequalities (LMI) formulation with parameter dependent Lyapunov functions. The results of the proposed synthesis is a gain scheduled controller that guarantees stability even for a nonlinear plant. In [23] a switched controller is proposed based on the inverse optimal theory. The obtained controller is then modified by gain scheduling to achieve the quality of the closed-loop system performance.

Two papers are representatives of the switched controller design for linear continuous time systems $[3,6]$. In [6] the authors introduce the notion of the dwelltime $T_{d}$ (minimal time interval between switching) into the switched controller design procedure. In the stability analysis condition of switched systems the dwell-time is included to the term $e^{A_{c} T_{d}}\left(A_{c}\right.$-closed-loop matrix (11)). In such a way the proposed design procedure for stability analysis and switching controller design for the real switching time interval $T>T_{d}$ becomes conservative. The "dwell-time term" for continuous-time systems very complicated the switched controller design procedure. In [3], sufficient conditions are given for the stability of linear systems with the dwell-time and with polytopic type parameter uncertainty. Lyapunov functions, in a quadratic form for each mode, which are non-increasing at the switching instants are assigned to each mode. During the dwell-time this function varies piecewise linearly

\footnotetext{
* Institute of Robotics and Cybernetics, Faculty of Electrical Engineering and Information Technology, Slovak University of Technology in Bratislava, Ilkovičova 3, 81219 Bratislava, vojtech.vesely@stuba.sk;

** Department of Signals and Systems, Chalmers University of Technology, Goteborg SE-412 96, Sweden, adrian.ilka@stuba.sk
} 
in time after switching occurs. The proposed method was applied to stabilization via state feedback for both nominal and uncertain cases. Since within the dwell-time the Lyapunov function varies piecewise linearly and the real switching time interval $T>T_{d}$, the switching controller design procedure becomes rather conservative. The switched controller design procedure for continuous-time systems proposed in this paper does not use the approach of the "dwell-time", therefore there is no such drawback as mentioned in the above references.

In this paper a design procedure is proposed for an LMI switched gain scheduled controller for continuous and discrete-time systems the based on a novel switched plant model for a class of nonlinear systems. The proposed design procedure is based on a special type of Lyapunov function. The procedure can be easily extended to the case of the robust switched gain scheduled controller design. A great many references on the switched controller design for continuous time systems focuss on the case where switching can occur immediately (ideal case), for a large number of switched systems the realistic case is where the rate of change of the switching signal is finite (non-ideal case). The assumption of a non-ideal case of the switching variable will be used in this note which gives other opportunities for the designer. Some results about the stability of a class of uncertain linear varying systems (transform to switched systems) can be found in [22].

Gain scheduling control is appealing to deal with systems subject to parametric variations, which include linear systems with time-varying parameters or nonlinear systems modeled as linear parameter varying systems [21]. In many applications, a gain scheduled controller must accommodate a plant with changing dynamics, where the dynamics is strongly dependent on the operating conditions. Reviews of the gain scheduled plant model can be found in $[8,13,26]$. Because of time varying gain scheduled parameters many researcher tackled the design problem of gain scheduled controllers for linear time-varying parameters system (LPV) using the linear matrix inequalities and the Lyapunov function approach $[1,18,21,28,30]$. In such cases, the designed gainscheduled controller must be able to stabilize and guarantee a reasonable performance for all operating conditions.

The remainder of the manuscript is organized as follows. In Section 2 we present the development of the gain scheduled plant model for a class of nonlinear switched systems and switched gain scheduled control systems considered and some preliminaries. In Section 3 we address the output feedback PI switched gain scheduled controller design procedure for continuous and discrete-time systems. Finally, in Section 4 the proposed design procedure is demonstrated on a simple example.

Our notations are standard, $D \in \mathbb{R}^{m \times n}$ denotes the set of real $m \times n$ matrices. $I_{m}$ is $m \times m$ identity matrix and $0_{m}$ denotes a zero matrix. If the size can be determined from the context, we will omit the subscript. $P>0$ $(P \geq 0)$ is a real symmetric, positive definite (semidefinite) matrix. $\sigma \in S$ indicates the arbitrary switching algorithm and $\sigma+1$ is the first next mode to mode $\sigma$ for switching system. $*$ in matrices denotes the respective transposed term to make the matrix symmetric.

\section{PROBLEM STATEMENT AND PRELIMINARIES}

\subsection{Gain scheduling model}

\section{Consider a family of nonlinear systems}

$$
\begin{aligned}
& \dot{z}=f_{\sigma}(z, v, w) \\
& \bar{y}=h(z)
\end{aligned}
$$

where $z \in \mathbb{R}^{n}$ is the state, the input $v \in \mathbb{R}^{m}$, the output $\bar{y} \in \mathbb{R}^{l}$, exogenous input $w \in \mathbb{R}^{k}$ which captures parametric dependence of the plant (1) on exogenous input (working point, outputs, and so on). The arbitrary switching algorithm $\sigma \in S=\{1,2, \ldots, N\}$ is a piecewise constant, right continuous function which specifies at each time the index of the active system [20]. Assume that for every $\sigma \in S, f_{\sigma}()$ is locally Lipschitz. A plant (1) equilibrium point $z_{e}, v_{e}, w_{e}$ is such a point that $f_{\sigma}\left(z_{e}, v_{e}, w_{e}\right)=0$. The following definition describes a set of equilibrium points which will be parameterized by the scheduling variable $\theta \in \mathbb{R}^{p}$

Definition 1 [26]. Functions $z_{e}, v_{e}, w_{e}$ define an equilibrium family for the plant (1) if

$$
f_{\sigma}\left(z_{e}, v_{e}, w_{e}\right)=0, \sigma \in S
$$

Associated with this equilibrium family is the measured output family

$$
\bar{y}_{e}=h\left(z_{e}\right) .
$$

Let us assume that the number of equilibrium points (working points) is equal to $p$, then corresponding to a specified $p$ equilibrium plant family there is a linearized plant family model for each plant mode $\sigma \in S p$ in the following form

$$
\begin{aligned}
& \dot{x}=\bar{A}_{\sigma i} x+\bar{B}_{\sigma i} u \\
& y=C x, i=1,2, \ldots, p, \sigma \in S=\{1,2, \ldots, N\}
\end{aligned}
$$

where $x=z-z_{e}, u=v-v_{e}, y=\bar{y}-\bar{y}_{e},\left(z_{e}, v_{e}, \bar{y}_{e}\right)$ define the equilibrium family for plant (1). Assume, that for $i$-th equilibrium point one obtain the sets $x \in X_{i}, u \in U_{i}$, $y \in Y_{i}, i=1,2, \ldots p$. Summarizing above sets we get $x \in X=\bigcup_{i=1}^{p} X_{i}, u \in U=\bigcup_{i=1}^{p} U_{i}, y \in Y=\bigcup_{i=1}^{p} Y_{i}$.

$$
\bar{A}_{\sigma i}=\frac{\partial f_{\sigma}}{\partial z}\left(z_{e i}, v_{e i}, w_{e i}\right), \quad \bar{B}_{\sigma i}=\frac{\partial f_{\sigma}}{\partial v}\left(\xi_{e i}, v_{e i}, w_{e i}\right)
$$


From (4) for each plant mode one obtains the gain scheduled plant model as a solution of the following linear equations

$$
\bar{A}_{\sigma i}=A_{\sigma o}+\sum_{j=1}^{p} A_{\sigma j} \theta_{j}, \quad i=1,2, \ldots p, \sigma=1,2, \ldots N
$$

where $\theta^{\top}=\left[\theta_{1} \ldots \theta_{p}\right] \in \Omega$ scheduling variable is treated as plant parameters which may be time varying and for which we assume that they are known, and lower and upper bounds are available for the parameters values and rate variations. Specifically:

- Each parameter $\theta_{j}$ ranges within the given interval

$$
\theta_{j} \in\left\langle\underline{\theta}_{j}, \bar{\theta}_{j}\right\rangle, j=1,2, \ldots p, \theta \in \Omega .
$$

- The rate of variation $\theta_{j}$ is well defined at all times and satisfies

$$
\dot{\theta}_{j} \in\left\langle\underline{\dot{\theta}}_{j}, \overline{\dot{\theta}}_{j}\right\rangle, \dot{\theta} \in \Omega_{t}
$$

To obtain the gain scheduled plant model, first $A_{\sigma 0}$ $\left(B_{\sigma 0}\right)$ is calculated as $A_{\sigma o}=\frac{1}{p} \sum_{i=1}^{p} \bar{A}_{\sigma i}$ and then from (5) one can obtain $A_{\sigma j}$ substituting the lower and upper bounds for $\theta_{j}$. Equations (4), (5) established a relationship between the nonlinear model (1) and corresponding family of linear ones (5), [13] and [26]. Note that due to (5) for each mode of the plant the obtained set of linear models (4) is retrieved by one gain scheduled plant model in the form

$$
\begin{aligned}
& \dot{x}=\left(A_{\sigma 0}+\sum_{j=1}^{p} A_{\sigma j} \theta_{j}\right) x+\left(B_{\sigma 0}+\sum_{j=1}^{p} B_{\sigma j} \theta_{j}\right) u, \\
& y=C x, \quad \sigma \in S, \quad \theta \in \Omega .
\end{aligned}
$$

For more details how to obtain the gain scheduled plant model, see $[8,13,26]$.

\subsection{Problem formulation and preliminaries}

For each plant mode of the switched gain scheduled LPV plant model the following system is considered

$$
\begin{aligned}
& \delta x(t)=\left(A_{\sigma 0}\right.\left.+\sum_{j=1}^{p} A_{\sigma j} \theta_{j}\right) x(t) \\
&+\left(B_{\sigma 0}+\sum_{j=1}^{p} B_{\sigma j} \theta_{j}\right) u(t) \\
& y(t)=C x(t), \quad \sigma \in S
\end{aligned}
$$

where

1) $\delta x(t)=\dot{x}(t)$ for continuous-time system and

2) $\delta x(t)=x(t+1)$ for discrete-time system.

For each switched plant mode the output feedback gain scheduled control law is considered in the form

$$
u(t)=\left(F_{\sigma 0}+\sum_{j=1}^{p} F_{\sigma j} \theta_{j}\right) y(t), \sigma \in S, \theta \in \Omega
$$

where $F_{\sigma k} \in \mathbb{R}^{m \times l}, k=0,1,2, \ldots p$ are constant output feedback gain matrices. For each plant mode the output feedback gain matrices can be structured in the decentralized, centralized and so on form. The closed-loop for the switched gain scheduled system is

$$
\delta x(t)=A_{c}(\theta, \alpha) x(t)
$$

where

$$
A_{c}(\theta, \alpha)=\sum_{\sigma=1}^{N} A_{\sigma}(\theta) \alpha_{\sigma}
$$

$$
\begin{aligned}
& A_{\sigma}(\theta)=A_{\sigma 0}+\sum_{j=1}^{p} A_{\sigma j} \theta_{j} \\
&+\left(B_{\sigma 0}+\sum_{j=1}^{p} B_{\sigma j} \theta_{j}\right)\left(F_{\sigma 0}+\sum_{j=1}^{p} F_{\sigma j} \theta_{j}\right) C, \\
& \alpha^{\top}=\left[\alpha_{1}, \ldots, \alpha_{N}\right], \quad \sum_{\sigma=1}^{N} \alpha_{\sigma}=1, \quad \sum_{\sigma=1}^{N} \dot{\alpha}_{\sigma}=0, \\
& \dot{\alpha}_{\sigma} \in\left\langle\underline{\dot{\alpha}}_{\sigma}, \overline{\dot{\alpha}}_{\sigma}\right\rangle \in \Omega_{d}, \quad \alpha_{\sigma} \in \Omega_{\alpha}, \quad \alpha_{\sigma} \geq 0
\end{aligned}
$$

and $\alpha_{j}=1$ when $\sigma_{j}$ is an active plant mode, else $\alpha_{j}=0$. Assume $\alpha \in \Omega_{\alpha}, \dot{\alpha} \in \Omega_{d}$.

To assess the system performance, we consider an original weighted scheduled quadratic cost function which allows to obtain the desired performance in the different plant equilibrium points for

- continuous-time system

$$
\begin{gathered}
J=\int_{t=0}^{\infty} J(t) \mathrm{d} t, \\
J(t)=x(t)^{\top} Q(\theta) x(t)+u^{\top}(t) R u(t),
\end{gathered}
$$

- discrete-time case

$$
\begin{gathered}
J_{d}=\sum_{t=0}^{\infty} J_{d}(t), \\
J_{d}(t)=x(t)^{\top} Q(\theta) x(t)+u^{\top}(t) R u(t)
\end{gathered}
$$

where $Q(\theta)=Q_{0}+\sum_{j=1}^{p} Q_{j} \theta_{j} \geq 0, R>0$.

Definition 2. Consider a stable closed-loop switched system (11) with $N$ modes. If there is a control law $u(t)$ (10) and a positive scalar $J^{*}$ such that the closed-loop cost function (13) or (14) satisfies $J \leq J^{*}\left(J_{d} \leq J^{*}\right)$ for all $\theta \in \Omega, \alpha \in \Omega_{\alpha}$ then $J^{*}$ is said to be a guaranteed cost and $u(t)$ is said to be a guaranteed cost control law for arbitrary switching system (11). 
Lemma 1 [12]. Control algorithm (10) is the guaranteed cost control law for the switched closed-loop system (11) if and only if there are a Lyapunov function $V(x(t), \theta(t), \alpha(t), t)$, matrices $Q(\theta), R$ and gain matrices $F_{\sigma k}, k=0,1,2, \ldots p, \sigma \in S$ such that the following inequality holds

$$
B_{e}(t)=\delta V(x(t), \theta(t), \alpha(t), t)+J(t)<0
$$

where

- $\delta V(x(t), \theta(t), \alpha(t), t)=\frac{d V(x(t), \theta(t), \alpha(t), t)}{d t}$

for continuous-time system,

- $\delta V(x(t), \theta(t), \alpha(t), t)=V(x(t+1), \theta(t+1), \alpha(t+1), t+$ $1)-V(x(t), \theta(t), \alpha(t), t)$ for discrete-time system.

We proceed with the notion of multi-convexity of a scalar quadratic function [5].

LEMma 2. Consider a scalar quadratic function of $\theta \in$ $R^{p}$

$$
f(\theta)=a_{0}+\sum_{j=1}^{p} a_{j} \theta_{j}+\sum_{j=1}^{p} \sum_{i>j}^{p} a_{j i} \theta_{i} \theta_{j}+\sum_{j=1}^{p} a_{j j} \theta_{j}^{2}
$$

and assume that if $f(\theta)$ is multi-convex, that is

$$
\frac{\partial^{2} f(\cdot)}{\partial \theta_{j}^{2}}=2 a_{j j} \geq 0, \quad j=1,2, \ldots p
$$

then $f(\theta)$ is negative in the hyper rectangle (6) if and only if it takes negative values at the vertices of (6), that is if and only if $f(\theta)<0$ for all vertices of the set given by $(6)$.

\section{MAIN RESULTS}

This section formulates the theoretical approach to the switched gain scheduled controller design with control law (10) which ensures closed-loop system multi parameterdependent quadratic stability and guaranteed cost for arbitrary switched algorithm $\sigma \in S$ for all gain scheduled parameters $\theta \in \Omega, \alpha \in \Omega_{\alpha}$ and $\dot{\theta} \in \Omega_{t}\left(\Delta \theta \in \Omega_{t d}\right)$, $\dot{\alpha} \in \Omega_{d}$.

\subsection{Design of switched gain scheduled controller: Continuous-time case}

Assume that in Lemma 1 the Lyapunov function is in the form

$$
V(x(t), \theta(t), \alpha(t), t)=x(t)^{\top} P(\theta, \alpha) x(t)
$$

where the novel Lyapunov matrix is

$$
P(\theta, \alpha)=P_{0}+\sum_{\sigma=1}^{N}\left(P_{\sigma 0}+\sum_{j=1}^{p} P_{\sigma j} \theta_{j}\right) \alpha_{\sigma}
$$

For time derivative of (17) one obtains

$$
\dot{V}(\cdot)=\left[\begin{array}{ll}
\dot{x}(t)^{\top} & x(t)^{\top}
\end{array}\right]\left[\begin{array}{cc}
0 & P(\theta, \alpha) \\
P(\theta, \alpha) & \dot{P}(\theta, \alpha)
\end{array}\right]\left[\begin{array}{c}
\dot{x}(t) \\
x(t)
\end{array}\right]
$$

where

$\dot{P}(\theta, \alpha)=\sum_{\sigma=1}^{N}\left(\sum_{\sigma=1}^{N} P_{\sigma 0} \dot{\alpha}_{\sigma}+\sum_{j=1}^{p} P_{\sigma j} \dot{\theta}_{j}+\sum_{j=1}^{p} \sum_{\sigma=1}^{N} P_{\sigma j} \dot{\alpha}_{\sigma} \theta_{j}\right) \alpha_{\sigma}$.

Using the equality

$$
\left(2 G_{1} \dot{x}+2 G_{2} x\right)^{\top}\left(\dot{x}-A_{c}(\theta, \alpha)\right)=0
$$

where $G_{1}, G_{2} \in R^{n \times n}$ are auxiliary matrices and summing (18) with (19) (after some manipulations) one obtains

$$
\frac{\mathrm{d} V(\cdot)}{\mathrm{d} t}=\sum_{\sigma=1}^{N}\left[\dot{x}(t)^{\top} \quad x(t)^{\top}\right] L_{\sigma}\left[\dot{x}(t)^{\top} \quad x(t)^{\top}\right]^{\top} \alpha_{\sigma}
$$

where

$$
\begin{aligned}
L_{\sigma}= & {\left[\begin{array}{cc}
L_{\sigma 11} & L_{\sigma 12} \\
* & L_{\sigma 22}
\end{array}\right], } \\
L_{\sigma 11}= & G_{1}^{\top}+G_{1}, \\
L_{\sigma 12}= & -G_{1}^{\top} A_{\sigma}+G_{2}+P_{0}+P_{\sigma 0}+\sum_{j=1}^{p} P_{\sigma j} \theta_{j}, \\
L_{\sigma 22}= & -G_{2}^{\top} A_{\sigma}-A_{\sigma}^{\top} G_{2}+\sum_{\sigma=1}^{N} P_{\sigma 0} \dot{\alpha}_{\sigma} \\
& +\sum_{j=1}^{p} P_{\sigma j} \dot{\theta}_{j}+\sum_{j=1}^{p} \sum_{\sigma=1}^{N} P_{\sigma j} \dot{\alpha}_{\sigma} \theta_{j}
\end{aligned}
$$

Note that if $\dot{V}(\cdot)<0$ or $L_{\sigma}<0$ (20) for all $\sigma \in S$, $\theta \in \Omega, \dot{\theta} \in \Omega_{t}, \dot{\alpha} \in \Omega_{d}$, the closed loop gain scheduled system is parameter-dependent quadratically stable for an arbitrary switching algorithm.

Substituting (10) to (13), after some manipulation one obtains

$$
J(t)=x(t)^{\top} N(t, \theta) x(t)
$$

where

$$
\begin{aligned}
N(t, \theta) & =N_{0}+\sum_{j=1}^{p} N_{j} \theta_{j}+\sum_{j=1}^{p} \sum_{k>j}^{p} N_{j k} \theta_{j} \theta_{k}+\sum_{k=1}^{p} N_{k k} \theta_{k}^{2} \\
N_{0} & =Q_{0}+C^{\top} F_{\sigma 0}^{\top} R F_{\sigma 0} C \\
N_{j} & =Q_{j}+C^{\top}\left(F_{\sigma j}^{\top} R F_{\sigma 0}+F_{\sigma 0}^{\top} R F_{\sigma j}\right) C \\
N_{j k} & =C^{\top}\left(F_{\sigma j}^{\top} R F_{\sigma k}+F_{\sigma k}^{\top} R F_{\sigma j}\right) C \\
N_{k k} & =C^{\top} F_{\sigma k}^{\top} R F_{\sigma k} C, \quad \sigma \in S .
\end{aligned}
$$


The model of $\sigma$ plant modes (12) can be rewritten to the form

$$
A_{\sigma}(\theta)=M_{0}+\sum_{j=1}^{p} M_{j} \theta_{j}+\sum_{j=1}^{p} \sum_{k>j}^{p} M_{j k} \theta_{j} \theta_{k}+\sum_{k=1}^{p} M_{k k} \theta_{k}^{2}
$$

where

$$
\begin{aligned}
M_{0} & =A_{\sigma 0}+B_{\sigma 0} F_{\sigma 0} C, \\
M_{j} & =A_{\sigma j}+\left(B_{\sigma 0} F_{\sigma j}+B_{\sigma j} F_{\sigma 0}\right) C, \\
M_{j k} & =\left(B_{\sigma j} F_{\sigma k}+B_{\sigma k} F_{\sigma j}\right) C, \\
M_{k k} & =B_{\sigma k} F_{\sigma k} C, \quad \sigma \in S .
\end{aligned}
$$

Substituting (20), (22) and (23) to Lemma 1 and due to Lemma 2 the closed-loop switched gain scheduled system with an arbitrarily switching algorithm is multi parameter-dependent quadratically stable if the following two inequalities hold

$$
\begin{gathered}
W_{0}+\sum_{j=1}^{p} W_{j} \theta_{j}+\sum_{j=1}^{p} \sum_{k>j}^{p} W_{j k} \theta_{j} \theta_{k}+\sum_{k=1}^{p} W_{k k} \theta_{k}^{2} \leq 0, \\
W_{k k} \geq 0, \quad \sigma \in S,
\end{gathered}
$$

where

$$
\begin{aligned}
W_{0}= & {\left[\begin{array}{cc}
W_{011} & W_{012} \\
* & W_{011}
\end{array}\right], } \\
W_{j}= & {\left[\begin{array}{ll}
0 & W_{j 12} \\
* & W_{j 22}
\end{array}\right], } \\
W_{j k}= & {\left[\begin{array}{cc}
0 & -G_{1}^{\top} M_{j k} \\
* & -G_{2}^{\top} M_{j k}-M_{j k}^{\top} G_{2}+N_{j k}
\end{array}\right], } \\
W_{k k}= & {\left[\begin{array}{cc}
0 & -G_{1}^{\top} M_{k k} \\
* & -G_{2}^{\top} M_{k k}-M_{k k}^{\top} G_{2}+N_{k k}
\end{array}\right], } \\
W_{011}= & G_{1}^{\top}+G_{1}, \quad \\
W_{012}= & -G_{1}^{\top} M_{0}+P_{0}+P_{\sigma 0}+G_{2}, \\
W_{022}= & -G_{2}^{\top} M_{0}-M_{0}^{\top} G_{2} \\
& +\sum_{\sigma=1}^{N} P_{\sigma 0} \dot{\alpha}_{\sigma}+\sum_{j=1}^{p} P_{\sigma j} \dot{\theta}_{j}+N_{0}, \\
W_{j 12}= & -G_{1}^{\top} M_{j}+P_{\sigma j}, \\
W_{j 22}= & -G_{2}^{\top} M_{j}-M_{j}^{\top} G_{2}+\sum_{\sigma=1}^{N} P_{\sigma j} \dot{\alpha}_{\sigma}+N_{j} .
\end{aligned}
$$

The main results of the switched gain scheduled controller design for a continuous-time system are given in the next theorem.

ThEOREM 1. The closed-loop continuous time system (11) with a switched gain scheduled controller (10) and an arbitrarily switching control algorithm is multi parameter-dependent quadratically stable with guaranteed cost for all $\sigma \in S, \theta \in \Omega, \dot{\theta} \in \Omega_{t}, \alpha \in \Omega_{\alpha}$ and $\dot{\alpha} \in \Omega_{d}$ if the inequalities (24) hold.

Proof. The proof of theorem sufficient condition is clear from previous considerations. Here, the proof is repeated only in basic steps. Substituting (20), (22) and (23) to Lemma 1 one obtains inequalities (24), which proves the sufficient conditions of Theorem 1 .

Note that the condition "if and only if" for a concrete structure of the Lyapunov matrix in Lemma 1 reduces to "if".

\subsection{Design of switched gain scheduled controller: Discrete-time case}

In this part of the note we assume that for discretetime systems the switching can occur immediately (ideal case), therefore with respect to switching variable $\sigma$ the quadratic stability approach will be used. Because of quadratic stability with respect to $\sigma$ let us assume that for discrete-time cases the Lyapunov function is in the form

$$
V(x(t), t, \theta(t), \sigma(t))=x(t)^{\top} P(\theta(t), \sigma(t)) x(t)
$$

The first difference of $(25)$ is

$$
\begin{aligned}
& \delta V(x(t), t, \theta(t), \sigma(t))=V(t+1, \theta(t+1), \sigma+1) \\
&-V(t, \theta(t), \sigma)=x(t+1)^{\top} P(\theta(t+1), \sigma+1) x(t+1) \\
&-x(t)^{\top} P(\theta(t), \sigma) x(t)
\end{aligned}
$$

where

$$
P(\theta(t), \sigma)=P_{\sigma 0}+\sum_{j=1}^{p} P_{\sigma j} \theta_{j}
$$

and $\sigma+1 \in S$ is the first next mode to mode $\sigma \in S$ for an arbitrary switching algorithm;

$$
P(\theta(t+1), \sigma+1)=P(\theta(t), \sigma+1)+\sum_{j=1}^{p} P_{\sigma+1 j} \Delta \theta_{j}(t)
$$

where $\Delta \theta(t)=\theta(t+1)-\theta(t) \in\langle\Delta \underline{\theta}, \Delta \bar{\theta}\rangle \in \Omega_{t d}$. After some manipulation for the first difference of the Lyapunov function (25) one obtains

$$
\delta V(\cdot)=\left[\begin{array}{ll}
x(t+1)^{\top} & x(t)^{\top}
\end{array}\right] L_{\sigma d}\left[x(t+1)^{\top} \quad x(t)^{\top}\right]^{\top}
$$

where

$$
\begin{aligned}
L_{\sigma d} & =\left[\begin{array}{cc}
L_{\sigma d 11} & 0 \\
0 & L_{\sigma d 22}
\end{array}\right], \\
L_{\sigma d 11} & =P_{\sigma+1_{0}}+\sum_{j=1}^{p} P_{\sigma+1_{j}}\left(\theta_{j}(t)+\Delta \theta_{j}(t)\right), \\
L_{\sigma d 22} & =P_{\sigma 0}+\sum_{j=1}^{p} P_{\sigma j} \theta_{j}(t) .
\end{aligned}
$$


Using the procedure given by equations. (19)-(23) for continuous-time systems the following parameter-dependent quadratic stability conditions with guaranteed cost and arbitrarily switching control algorithm for the design of a discrete-time switched gain scheduled controller are obtained

$$
\begin{aligned}
& V_{0}+\sum_{j=1}^{p} V_{j} \theta_{j}+\sum_{j=1}^{p} \sum_{k>j}^{p} V_{j k} \theta_{j} \theta_{k}+\sum_{k=1}^{p} V_{k k} \theta_{k}^{2}<0, \\
& V_{k k} \geq 0
\end{aligned}
$$

for all $\sigma \in S$ where

$$
\begin{aligned}
V_{0} & =\left[\begin{array}{cc}
V_{011} & V_{012} \\
* & V_{022}
\end{array}\right], \\
V_{j} & =\left[\begin{array}{cc}
V_{j 11} & V_{j 12} \\
* & V_{j 22}
\end{array}\right], \\
V_{j k} & =\left[\begin{array}{cc}
0 & -G_{1}^{\top} M_{j k} \\
* & -G_{2}^{\top} M_{j k}-M_{j k}^{\top} G_{2}+N_{j k}
\end{array}\right], \\
V_{k k} & =\left[\begin{array}{cc}
0 & -G_{1}^{\top} M_{k k} \\
* & -G_{2}^{\top} M_{k k}-M_{k k}^{\top} N_{G}+N_{k k}
\end{array}\right], \\
V_{011} & =G_{1}^{\top}+G_{1}+P_{\sigma+1_{0}}+\sum_{j=1}^{p} P_{\sigma+1_{j}} \Delta \theta_{j}(t), \\
V_{012} & =-G_{1}^{\top} M_{0}+G_{2}, \\
V_{022} & =-G_{2}^{\top} M_{0}-M_{0}^{\top} G_{2}+N_{0}+P_{\sigma 0}, \\
V_{j 11} & =P_{\sigma+1_{j}}, \\
V_{j 12} & =-G_{1}^{\top} M_{j}, \\
V_{j 22} & =-G_{2}^{\top} M_{j}-M_{j}^{\top} G_{2}+N_{j}+P_{\sigma j}, \\
V_{j k 12} & =-G_{1}^{\top} M_{j k}, \\
V_{j k 22} & =-G_{2}^{\top} M_{j k}-M_{j k}^{\top} G_{2}+N_{j k}, \\
V_{k k 12} & =-G_{1}^{\top} M_{k k}, \\
V_{k k 22} & =-G_{2}^{\top} M_{k k}-M_{k k}^{\top} G_{2}+N_{k k} .
\end{aligned}
$$

ThEOREM 2. The closed-loop discrete-time system (11) with switched gain scheduled controller (10) and an arbitrarily switching control algorithm is parameterdependent quadratically stable with guaranteed cost for all $\sigma \in S, \theta \in \Omega, \Delta \theta \in \Omega_{t d}$ if inequalities (28) hold for all vertices defined by (6) and (28).

Pr o o f . Proof of the theorem sufficient condition is clear from the previous consideration. Here, the proof is repeated only in basic steps. Substituting (22), (23) and (27) to Lemma 1 one obtains inequalities (28), which proves the sufficient conditions of Theorem 2 .

\section{NUMERICAL EXAMPLE}

In this section the proposed design procedure is applied to a continuous-time system to design a switched gain scheduled controller. The problem is to design a PI switched gain scheduled controller for a second order nonlinear switched continuous-time system with two modes and an arbitrary switching algorithm to guarantee the multi parameter-dependent quadratic stability and guaranteed value of a given structured cost function.

Consider the switched gain scheduled system (9) with two modes and two gain scheduled parameters. For the first and second plant modes the gain scheduled extended plant model [31] due to PI controller is as

First mode

$$
\begin{aligned}
& A_{10}=\left[\begin{array}{ccc}
-.5 & 0.2 & 0 \\
0.35 & -0.1 & 0 \\
1 & 0 & 0
\end{array}\right], \quad A_{11}=\left[\begin{array}{ccc}
-0.01 & -0.02 & 0 \\
0.035 & 0.02 & 0 \\
0 & 0 & 0
\end{array}\right] \text {, } \\
& A_{12}=\left[\begin{array}{ccc}
-0.05 & 0.02 & 0 \\
0.02 & 0.04 & 0 \\
0 & 0 & 0
\end{array}\right], \quad \begin{array}{ll}
B_{10}^{\top}=\left[\begin{array}{lll}
2 & 1 & 0
\end{array}\right], \\
B_{11}^{\top}=\left[\begin{array}{lll}
.2 & 0.5 & 0
\end{array}\right], \\
B_{12}^{\top}=\left[\begin{array}{lll}
0.01 & 0.03 & 0
\end{array}\right],
\end{array} \\
& C=\left[\begin{array}{lll}
1 & 0 & 0 \\
0 & 0 & 1
\end{array}\right] \text {. }
\end{aligned}
$$

Second mode

$$
\begin{aligned}
& A_{10}=\left[\begin{array}{ccc}
-.7 & 0.5 & 0 \\
0.6 & -0.3 & 0 \\
1 & 0 & 0
\end{array}\right], \quad A_{11}=\left[\begin{array}{ccc}
0.01 & -0.005 & 0 \\
0.02 & -0.05 & 0 \\
0 & 0 & 0
\end{array}\right] \\
& A_{12}=\left[\begin{array}{ccc}
0.001 & -0.03 & 0 \\
0.02 & -0.04 & 0 \\
0 & 0 & 0
\end{array}\right], \quad \begin{array}{ll}
B_{10}^{\top}=\left[\begin{array}{lll}
3 & 1 & 0
\end{array}\right], \\
B_{11}^{\top}=\left[\begin{array}{lll}
.02 & 0.02 & 0
\end{array}\right], \\
B_{12}^{\top}=\left[\begin{array}{lll}
0.02 & 0.05 & 0
\end{array}\right],
\end{array} \\
& C=\left[\begin{array}{lll}
1 & 0 & 0 \\
0 & 0 & 1
\end{array}\right] \text {. }
\end{aligned}
$$

Parameters of the cost function (13) are $R=r I_{r}$, $r=1, Q(\theta)=Q_{0}+Q_{1} \theta_{1}+Q_{2} \theta_{2}$ (in the term $Q(\theta)$ the gain scheduled parameter $\theta$ serves to ensure the desired performance in different plant working points) where $Q_{i}=q_{i} I, i=0,1,2, q_{0}=0.1, q_{1}=0.02, q_{2}=0.02$, for the Lyapunov matrix it holds $0<P(\theta, \sigma)<$ ro $I$, ro $=1000, \theta_{i} \in\langle-1,1\rangle, \dot{\theta}_{i} \in\langle-4,4\rangle, i=1,2$ and the rate of mode changes (18) $\dot{\alpha}_{i} \in\langle-200,200\rangle$. For the above parameters as the solution of (25) the following gain scheduled controllers are obtained:

First plant mode gain scheduled controller (10)

$$
\begin{gathered}
F_{10}=-1.2426-\frac{0.4353}{s} ; \quad F_{11}=0.657+\frac{0.1033}{s} \\
F_{12}=\left(0.6195+\frac{0.5741}{s}\right) 10^{-6}
\end{gathered}
$$

Second plant mode gain scheduled controller (10)

$$
\begin{gathered}
F_{20}=-0.8843-\frac{0.2914}{s} ; \quad F_{21}=\left(0.4355-\frac{0.3481}{s}\right) 10^{-7} \\
F_{22}=\left(0.1460+\frac{0.2294}{s}\right) 10^{-3}
\end{gathered}
$$



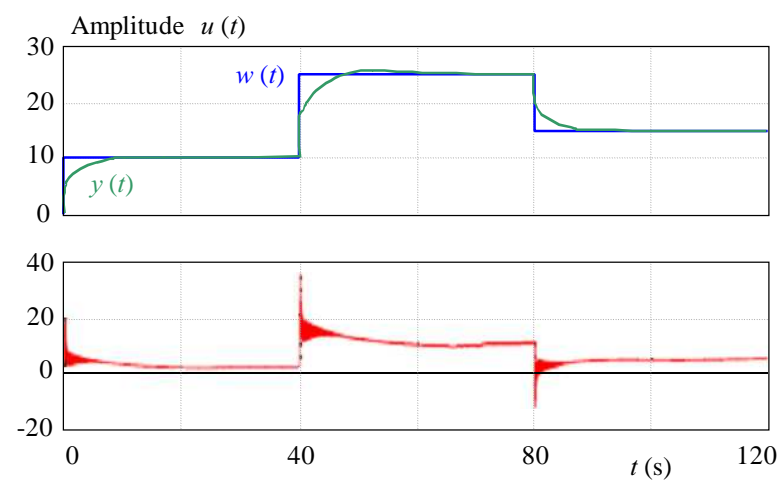

Fig. 1. Simulation results of output and input when $\dot{\theta}(t) \in\langle-4,4\rangle$, $\dot{\alpha} \in\langle-200,200\rangle$

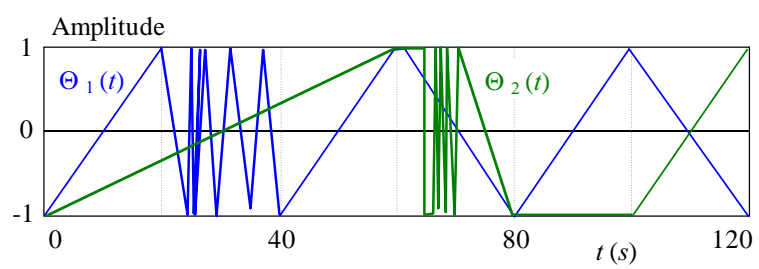

Fig. 2. Development of the gain scheduled parameters (working point changes) $\dot{\theta}(t) \in\langle-4,4\rangle$ during simulation

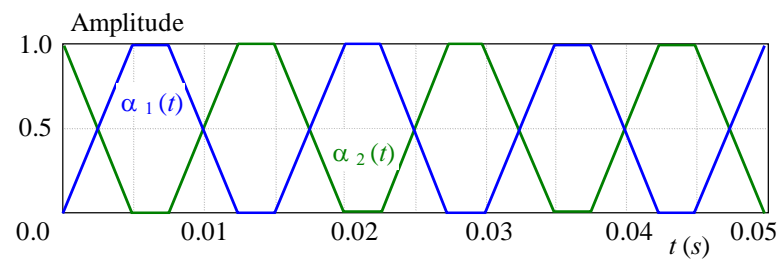

Fig. 3. Detail of two non-ideal switching parameters development when $\dot{\alpha} \in\langle-200,200\rangle$ during simulation

Note that the gain scheduled controller is in the form

$$
\operatorname{Controller}_{i}(s)=F_{i 0}+F_{i 1} \theta_{1}+F_{i 2} \theta_{2}, \quad i=1,2 \text {. }
$$

The maximal eigenvalue of the closed-loop switched system for the case of $\theta_{1}=\theta_{2}=0$ is -0.2471 . The maximum rate of change of value (18) is about $\dot{\alpha}_{\max } \in$ $\langle-250,250\rangle$. For a larger value of $\dot{\alpha}$ the gain scheduled controller design procedure is not feasible. Dynamic behaviors of the closed-loop switched gain scheduled system are given in Figs. 1, 2 and 3.

$\mathrm{R}$ e $\mathrm{m}$ ar $\mathrm{k} 1$. For decreasing the calculation loads of the design procedure for (17) one could choose the following inequalities

$$
0<P_{c}<\operatorname{ro} I, \quad 0<P_{\sigma j}<r o_{\sigma j} I
$$

then in (24) substitute to $W_{0}, W_{j}, V_{0}$ the following inequalities

$$
P_{\sigma j} \dot{\alpha}_{\sigma} \leq P_{\sigma j} \overline{\dot{\alpha}}_{\sigma}, \quad \dot{\theta}_{j} \leq \overline{\dot{\theta}}_{j}, \quad \Delta \theta(t) \leq \Delta \bar{\theta}_{j}
$$

$\mathrm{R}$ e $\mathrm{m}$ ark 2 . To assess the system performance we consider an original weighted scheduled quadratic cost function which allows to obtain the desired (different or the same) performance in the different plant equilibrium points.

R e m a r k 3 . In this example due to (12) for nonideal switching variables it holds $\alpha_{1}+\alpha_{2}=1, \dot{\alpha}_{1}+\dot{\alpha}_{2}=$ 0 . Time developments of the switching variables are given in Fig. 3.

\section{CONCLUSION}

A new method for the stability analysis and design of a switched controller for non-linear system using the gain scheduling approach for continuous and discretetime systems was introduced. In this paper the novel switched plant model for a class of nonlinear system was proposed.

The proposed switched controller design procedure is based on a novel plant model, a special type of the Lyapunov function and the Lyapunov function stability theory. For a continuous-time switched system the gain scheduled controller design procedure ensures multi parameter-dependent quadratic stability and guaranteed cost for an arbitrary switching law and a finite (infinite) rate of switching signal change. The switched controller design procedure for continuous-time systems proposed in this paper does not use the approach of the "dwelltime" which very complicated the switched controller design procedure. In this note, we introduce the assumption of a non-ideal case of the switching variable (rate of switching variable is finite) which gives other opportunities for the designer. The working plant parameters may change very rapidly but the rate of parameters change are bounded ( $\overline{\dot{\theta}}_{i}$ is bounded). For discrete-time switched systems due to the novel plant a new design procedure is obtained which ensures parameter-dependent quadratic stability and guaranteed cost for an arbitrary switching law and for a given finite rate of change of the plant parameters.

To guarantee the desired system performance, in this paper we consider an original weighted scheduled quadratic cost function which allows to obtain the desired performance in different equilibrium points of the plant. The designed design procedure can easily be extended to the case of a robust switched gain scheduled controller using the idea of paper [27]. The numerical example illustrates the effectiveness of the proposed approach.

\section{Acknowledgement}

The work has been supported by the Slovak Scientific Grant Agency, Grant No 1/0475/16. 


\section{REFERENCES}

[1] AdEgas, F. D.-STOUstrup, J.: Structured Control of Affine Linear Parametric Varying Systems, In: ACC San Francisco, June 2011, pp. 739-744.

[2] AleKSANDrov, A. Yu.-CHEN, Y.-PLATONOV, A. V.ZHANG, L. : Stability Analysis for a Class of Switched Nonlinear Systems, Automatica 47 (2011), 2286-2291.

[3] ALlEWRHAND, L. I.-SHAKED, U.: Robust Stability and Stabilization of Linear Switched Systems with Dwell Time, IEEE Trans on AC 56 (2011), 381-386.

[4] BRANICKY, M. S.-BORKAR, V. S.-MITTER, S. K.: A Unified Framework for Hybrid Control: Model and Optimal Control Theory, IEEE Transactions on Automatic Control 43 No. 1 (1998), 31-45.

[5] Gahinet, P.-APKARian, P.-Chilali, M.: Affine Parameter-Dependent Lyapunov Functions and Real Parametric Uncertainty, IEEE Trans on AC 41 No. 3 (1996), 436-442.

[6] Geromel, J. C.-COLONERI, P.: Stability and Stabilization of Continuous Time Switched Linear Systems, SIAM J. Control Optim. 45 No. 5 (2006), 1915-1930.

[7] GOEBEL, R.-TEEL, A. R.: Solutions to Hybrid Inclusions via Set and Graphical Convergence with Stability Theory Applications, Automatica 42 No. 4 (2006), 573-587.

[8] GUOLIANG WEI-ZIDONG WANG-WANGYAN LI-LIFENG MA : A Survey on Gain-Scheduled Control and Filtering for Parameter-Varying Systems, Discrete Dynamics in Nature and Sociaty, vol. 2014, Article ID 105815.

[9] SHAKER, H. R.-HOW, J. P.: Stability Analysis for a Class of Switched Nonlinear Systems, In: ACC, 2010, pp. 2517-2520.

[10] HANIFZADEGAN, M.-NAGAMUNE, R.: Switching Gain Scheduled Control Design for Flexible Ball-Srew Drives, Journal of dynamic systems, measurement and control 136 No. 1 (2013).

[11] HORRI, N.-PALMER, P.-ROBERTS, M.: Gain Scheduled Inverse Optimal Satelit Attitude Control, IEEE trans. on Aerospace and Electronic Systems 48 No. 3 (2012), 2437-2457.

[12] KUNCEVIC, V. M.-LYCAK, M. M.: Control Systems Design using Lyapunov Function Approach, Nauka, Moscow, 1977. (in Russian)

[13] LEITH, D. J.-LEITHEAD, W. E. : Survey of Gain Scheduling Analysis and Design, Inter. Journal of Control 73 No. 11 (2000), 1001-1025.

[14] LIJUN LONG - JUN ZHAO : Global Stabilization for a Class of Switched Nonlinear Feedforward Systems, Systems and Control Letters 60 (2011), 734-738.

[15] LIBERZON, D. : Switching in Systems and Control, Birkhauser, Boston, 2003.

[16] LUNZE, J.-LAGARRIGUE, F. L. Editors: Hanbook of Hybrid Systems Control, Theory, Tools, Applications, Cambridge University Press, 2009.

[17] LYGEROS, J.: On Overview of Research Areas in Hybrid Systems Control, In: Proc. of the 44 IEEE Conf. on Decission and Control and the European Control Conf., Spain, 2005.

[18] MASAYUKI SATO-DIMITRI PEAUCELLE: Gain Scheduled Output Feedback Controllers using Inexat Scheduling Parameters for Continuous-Time Varying Systems, Automatica 49 (2013), 1019-1025.

[19] MASIH HAMIFZADEGAN-RYOZO NAGAMUNE: Switching Gain scheduled Control Design for Flexible Ball-Screw Drives, J. Dyn. Sys. Meas. Control 136 No. 1 (2013).
[20] MUlleR, M. A.-LIBERZON, D.: Input/Output-to State Stability of Switched Nonlinear Systems, In: ACC, June 2010, pp. $1708-1712$.

21] MOnTAGneR, V. F.-PERES, P. L. D. : State Feedback Gain Scheduling for Linear Systems with Time-Varying Parameters, In: ACC Boston, 2004, pp. 2004-2009.

[22] MONTAGneR, V.-PERES, P. L. D. : A New LMI Condition for the Robust Stability of Linear Time-Varying Systems, In: Proceedings of CDC, 2003, pp. 6133-6138.

[23] HORRI, N. M.-PALMER, P.-ROBERTS, M. : Gain-Scheduled Inverse Optimal Satellite Attitude Control, Trans. on Aerospace and Electronic Systems 48 (2011), 2437-2457.

24] El-FARR, N. H.-PRASHANT MHASKAR-CHRISTOFIDES, P. D.: Output Feedback Control of Switched Nonlinear Systems using Multiple Lyapunov Functions, Systems and Control Letters 54 (2005), 1163-1182.

[25] OLALLA, C.-LEYVE, R.-QUEINNEC, I.-MAKSIMOVIC, D. : Robust Gain-Scheduled Control of Switched-Mode DC-DC Convertors, IEEE Trans on Power Elecrtonics 27 No. 6 (2012), 3006-3019.

[26] RUGH, W. J.-SHAMMA, J. S.: Survey Research on Gain Scheduling, Automatica 36 No. 10, 1401-1425.

[27] VESELÝ, V.-ILKA, A.: PID Robust Gain-Scheduled Controller Designinbook In: ECC'2014, June 24-25, 2014, Strasbourg, France, CD-ROM.

28] VESELÝ, V.--ILKA, A.: Gain-Scheduled PID Controller Design, Journal of Proces Control 23 (2013), 1141-1148.

29] YUANGONG SUN-LONG WANG: On Stability of Switched Nonlinear Systems, Automatica 49 (2013), 305-307.

30] WADA, N.-SAEKI, M. : An LMI based Scheduling Algorithm for Constrained Stabilization Problem, Systems and Control Letters 57 (2008), 255-261.

[31] VESELÝ, V.-ROSINOVÁ, D. : Robust PID-PSD Controller Design: BMI Approach, Asian Journal of Control 15 No. 2 (2013), 469-478.

Received 15 December 2015

Vojtech Veselý was born in Velké Kapušany, Slovakia in 1940. He received MSc degree in Electrical Engineering from the Leningrad Electrical Engineering Institute, St. Peterburg, Russia, in 1964, PhD and DSc degrees from the Slovak University of Technology, Bratislava, Slovak Republic, in 1971 and 1985, respectively. Since 1964 he has been with the Department of Automatic Control Systems, STU FEI in Bratislava. Since 1986 he has been a full professor. His research interests include the areas of power system control, decentralized control of large-scale systems, robust control, predictive control and optimization. He is author or coauthor of more than 300 scientific papers.

Adrian Ilka was born in Dunajská Streda, Slovakia in 1987. He received BSc degree from the Faculty of Electrical Engineering and Information Technology, Slovak University of Technology in Bratislava in 2010 and MSc and in 2014 $\mathrm{PhD}$ degree in technical cybernetics. He is interested in power system control, robust control, Lyapunov theory of stability, linear matrix inequalities and gain-scheduled control. 